\title{
Lidil
}

Revue de linguistique et de didactique des langues

$59 \mid 2019$

Prononcer les langues : variations, émotions, médiations

\section{Maude Vadot, Françoise Roche et Chahrazed Dahou (dir.), Genre et sciences du langage : enjeux et perspective}

Montpellier, Presses universitaires de la Méditerranée, 2017, 314 p.

Nolwenn Lorenzi Bailly

\section{OpenEdition}

Journals

Édition électronique

URL : http://journals.openedition.org/lidil/5954

DOI : 10.4000/lidil.5954

ISSN : 1960-6052

Éditeur

UGA Éditions/Université Grenoble Alpes

Édition imprimée

ISBN : 978-2-37747-090-7

ISSN : 1146-6480

Référence électronique

Nolwenn Lorenzi Bailly, « Maude Vadot, Françoise Roche et Chahrazed Dahou (dir.), Genre et sciences du langage : enjeux et perspective », Lidil [En ligne], 59 | 2019, mis en ligne le 01 mai 2019, consulté le 24 septembre 2020. URL : http://journals.openedition.org/lidil/5954; DOI : https://doi.org/10.4000/lidil. 5954

Ce document a été généré automatiquement le 24 septembre 2020.

(C) Lidil 


\section{Maude Vadot, Françoise Roche et Chahrazed Dahou (dir.), Genre et sciences du langage : enjeux et perspective}

Montpellier, Presses universitaires de la Méditerranée, 2017, 314 p.

Nolwenn Lorenzi Bailly

\section{RÉFÉRENCE}

Maude Vadot, Françoise Roche et Chahrazed Dahou (dir.), Genre et sciences du langage : enjeux et perspective, Montpellier, Presses universitaires de la Méditerranée, 2017, 314 p.

1 Les études de genre, apparues dans un premier temps aux États-Unis sous la nomination de Gender Studies s'imposent petit à petit dans le champ des sciences sociales et peut-être plus spécifiquement au cœur des sciences du langage, ne serait-ce que parce que le genre questionne les façons de dire. Ainsi en est-il du débat sur l'écriture inclusive, l'écriture féminine ou encore «l'écriture corps » (Boustani, 1993). L'imposition et la construction d'identités genrées se créent par le langage. C'est en effet parce que le genre masculin a été considéré comme le plus noble que la règle grammaticale impose un accord au masculin qui l'emporte sur le féminin. Cette idée se développe à partir du XVII ${ }^{\mathrm{e}}$ siècle par Vaugelas notamment, puis un siècle plus tard par Dupleix ou encore Beauzée qui rappelle que «le genre masculin est réputé plus noble que le féminin à cause de la supériorité du mâle sur la femelle ». Les impositions genrées rendent compte des langues, de leurs utilisations et des visions du monde. Il y a d'abord ce « rapport social hiérarchique divisant le monde en deux catégories fondées sur des caractéristiques sexuelles» (p.11). Ainsi le rappelle Anne-Marie Houdebine (p. 287), «si tous et toutes discutent, seules les femmes bavardent» (Aebisher, 1983) preuve d'une imposition linguistique identitaire. Mais ne considérer que deux 
catégories est ici encore imposer une domination à l'encontre de celles et ceux qui refusent d'être genrée's. Ainsi, le genre doit être questionné et c'est ce que propose l'ouvrage en débutant par une épistémologie et une histoire des études du genre. En convoquant les travaux d'Edwar Sapir, Véronique Perry interroge les catégories conceptuelles, linguistiques, sociales et sexuelles pour, respectivement «penser, désigner, identifier [et] normer l'humain» (p.21) et envisage le lien entre aspects formels et aspects sociaux de la problématique. En effet, Véronique Perry rappelle que la métaphorisation du genre en fait « une forme de "diktat binaire" [...] qui relève d'une interprétation ethnocentrique des sexes et de l'organisation socio-symbolique qui en découle dans la langue et le social» (p. 30). L'auteure pense donc, à l'instar d'Andrea d'Urso, que la catégorisation du genre est idéologique et fait apparaitre un «mode de production domestique [permettant de] réaliser un projet de société patriarcale» (p. 47). Andrea d'Urso propose un prolongement critique de la réflexion rossi-landienne pour son analyse. Lucy Michel quant à elle, questionne la notion de «masculin générique » se demandant d'ailleurs si l'on peut envisager un "féminin générique » en définissant dans un premier temps la notion de "valeur générique » attribuée au masculin, une notion qui ne va pas de soi.

2 Le lien entre genre et discours, parole, sciences du langage en général est questionné ainsi tout au long de l'ouvrage et est peut-être central au cœur de l'article de Maria Candea qui se propose d'interroger les apports du genre sur la perception de la parole à travers le prisme des stéréotypes. Via une enquête, Maria Candea (avec Aron Arnold, 2015) dévoile des stéréotypes assez «bien installés " «qui font que les voix aigües et claires [...] sont associées à la soumission [...] et en plus, pour les voix masculines, à l'homosexualité (Gaudio, 1994) » (p. 86). Si l'auteure souligne que certains écueils sont à éviter, n'en demeure que le genre rend compte de résistances idéologiques profondes.

La deuxième partie de l'ouvrage est orientée sur les pratiques discursives à l'instar de Sylvie Dubois et Natacha Jeudy qui traitent des éloges funèbres monastiques, Magali Guaresi qui analyse le discours électoral, et plus spécifiquement les professions de foi des élu·e's. C'est le réseau social Twitter qui a inspiré l'analyse de Camille LagardeBelleville et Michel Otell en partant plus particulièrement du «\#TweetCommeUnMec/ UneFille", tweet auquel viennent se greffer des considérations de genre. On remarquera par ailleurs que ce n'est pas « garçon » qui a été utilisé, en regard du terme " fille ", mais bien le substantif mec qui induit là encore une lecture de domination. Alice Coutant, enfin, rend compte des processus d'identification de soi et de l'autre au cœur des représentations binaires imposées par la société.

L'ouvrage se termine par une troisième partie qui met à l'honneur l'analyse du genre en regard de la didactique. Les chercheures y pointent du doigt l'importance d'une vigilance accrue dans l'éducation des enfants. Si aujourd'hui les dessins animés modernes n'hésitent plus à faire de leurs héroïnes de braves aventurières, cela ne reste qu'une minorité face à de belles endormies qui endossent le rôle d'infirmières aux côtés de preux chevaliers, policiers ou pompiers. Des modèles identificatoires qui gagneraient à être développés. Anne-Marie Houdebine peint avec intérêt la domination masculine là où déjà au début $\mathrm{du} \mathrm{xVIII}^{\mathrm{e}}$ siècle, les Précieuses travaillaient, avec le soutien de Poulain de la Barre, en faveur d'un accès des femmes à tous les enseignements et à tous les métiers, lesquels devraient parailleurs voir leur désignation féminisée. Quant à Mireille Baurens, elle fait le lien entre genre, qui «s'il n'est pas toujours visible il est toujours présent » (Sunderland, 2000) et didactique des 
langues-cultures, didactique présentée comme «un art du questionnement permanent» (Galisson \& Puren, 1999). Émilie Lebreton questionne la norme dans l'enseignement de la langue française, cette norme par ailleurs tant attendue par les élèves, ici des femmes migrantes. Ces attentes demandent d'interroger les pratiques de formation, ce que fait l'auteure en discutant, au cœur de ces pratiques, de la place et des enjeux de la langue normée. Ce ne sont pas les étudiante-s mais les manuels qui intéressent enfin Grâce Ranchon, manuel, qui, le rappelle-t-elle, demeure l'outil premier de l'enseignante (de FLE). C'est ainsi le concept d'hétéronormativité que l'auteure questionne en démontrant que les outils pédagogiques ne sont pas neutres, loin de là. Le dernier article est écrit par Maude Vadot et fait état des recherches de sa thèse de doctorat, recherches en lien avec les deux contributions précédentes puisqu'il s'agit d'analyser discours institutionnels et manuels à destination de personnes migrantes, l'auteure mettant en exergue, une fois de plus, des représentation stéréotypées et inégalitaires. On vous laisse deviner le genre dominant... La langue transporte les représentations qui vont parfois jusqu'à l'ordre du mépris (Yagello, 1978).

5 Toujours est-il que si avancées il y a, le débat est loin d'être clôt et renvoie à de nombreuses questions sociales. Ainsi, Marcel Kuntz, chercheur en biotechnologie au CNRS s'offusque sur l'utilisation grandissante de l'écriture inclusive rappelant les dires de l'Académie française et soulignant que « la langue française se trouve désormais en péril mortel» (Le Figaro, 5 février 2019). Ainsi conclurai-je de la même façon que l'ouvrage en affirmant que la lutte féministe a toujours sa raison d'être et que les sciences du langage doivent continuer à dénoncer les rapports de domination qui ne cessent de se manifester dans la langue. Joan Scott (1988, p. 59) l'affirmait déjà : « [...] le genre est une façon première de signifier les rapports de pouvoir. »

\section{AUTEURS}

\section{NOLWENN LORENZI BAILLY}

LIDILEM, Université Grenoble Alpes 